\title{
PRODUCTS WITH CLOSED PROJECTIONS
}

\author{
BY \\ N. NOBLE
}

Introduction. In this paper we study pairs $(X, Y)$ of topological spaces which have the property that one or both of the projections from $X \times Y$ is closed (maps closed sets to closed sets) or $z$-closed (maps zero sets to closed sets). These conditions on projections arise naturally from considerations involving the exponential map on function spaces; in $\S 1$ we make explicit their relationship to the exponential map by proving several "exponential laws." As applications of one of these, we give conditions necessary and sufficient that a product, or semiuniform product, of two fine spaces be fine.

The second and third sections are devoted to a study of conditions under which projections will be closed or $z$-closed. The results of the third section, together with the characterization of fine products in the first section, give a new, and entirely topological, proof of Isbell's characterization of (finite) fine products, Theorems 32, 33, and 35 in Chapter VII of [6]. They also give a new characterization of the $C^{*}$-pairs defined in [2].

I would like to thank A. W. Hager and W. W. Comfort for several helpful discussions concerning the results in this paper.

1. The exponential map. Let $F(X, Z)$ denote the set of functions from $X$ to $Z$, $C(X, Z)$ the continuous functions. Adopting the usual convention, we let $C(X)$ denote $C(X, R), R$ the real line. The map $\mu: F(X \times Y, Z) \rightarrow F(X, F(Y, Z))$ defined by the relation $(\mu f)(x)(y)=f(x, y)$ is called the exponential map. Where $\mathscr{T}$ is a topology for $C(Y, Z),(X, Y, Z, \mathscr{T})$ is said to satisfy the exponential law if $\mu$ maps $C(X \times Y, Z)$ onto $C(X, C(Y, Z))$.

We will define a class of topologies for $C(X, Z)$ by means of certain conditions on the graphs of the functions in $C(X, Z)$. Since it is common practice, when they are defined at all, to define a function as being its graph, we will not distinguish between these two notions. For a subset $S$ of $X \times Z$ let $N(S)=\{f \in C(X, Z): f \cap S$ $=\varnothing\}$. Let $\gamma_{a}$ be a function (from $C(X, Z)$ to $2^{\left(2^{X \times Z)}\right)}$ which associates with each $f$ in $C(X, Z)$ a collection $\gamma_{a}(f)$ of subsets $X \times Z$ so that for each $S$ in $\gamma_{a}(f), f \cap S=\varnothing$. The topology on $C(X, Z)$ which has $\left\{N(S): S \in \gamma_{a}(f)\right\}$ as a subbase for the neighborhood system at $f$, for each $f$, will be denoted as $\mathscr{T}_{a}$. In general the sets $N(S)$ will not be open. However, if $S \subseteq X \times Z$ is in $\gamma_{a}(f)$ whenever $f \cap S=\varnothing, N(S)$ is open. Thus if $\gamma_{a}^{*}$ is any collection of subsets of $X \times Z$ and we define $\gamma_{a}$ by the rule $\gamma_{a}(f)$ $=\left\{S \in \gamma_{a}^{*}: f \cap S=\varnothing\right\}$, the topology $\mathscr{T}_{a}$ will have subbase $\left\{N(S): S \in \gamma_{a}^{*}\right\}$. When

Received by the editors February 14, 1968. 
possible, we will define a topology $\mathscr{T}_{a}$ by listing $\gamma_{a}^{*}$, rather than $\gamma_{a}$. We will speak of $\mathscr{T}_{a}$ as being generated by $\gamma_{a}$, or, when applicable, by $\gamma_{a}^{*}$.

The class of topologies generated by sets $\gamma_{a}^{*}$ include all of the set-open topologies and is studied in [10]. A class of function space topologies which includes these and many of the hyperspace topologies is studied in [9]. The class of topologies generated by functions $\gamma_{a}$ includes, besides those generated by sets $\gamma_{a}^{*}$, the topologies of uniform convergence on members of a cover.

It is not our intention to study this class of topologies-our primary purpose in introducing it is to allow the following theorem to be stated in generality sufficient to indicate the importance of closure conditions on projections to a study of the exponential laws. Hopefully the applications in this section will indicate the importance of the exponential laws themselves. Before stating our first theorem we need one additional bit of notation: For a function $f: X \times Y \rightarrow Z$ let $g_{f}: X \times Y$ $\rightarrow Y \times Z$ be the function $g_{f}(x, y)=(y, f(x, y))$. Note that, since its compositions with the projections on $Y \times Z$ are $f$ and the identity map, $g_{f}$ is continuous if and only if $f$ is continuous.

1.1. TheOREM. Let $C(Y, Z)$ have the topology generated by $\gamma_{a}$. Then $\mu(C(X \times Y, Z))$ is contained in $C(X, C(Y, Z))$ if and only if for each $f$ in $C(X \times Y, Z)$, each $x_{0}$ in $X$, and each $S$ in $\gamma_{a}\left(\mu f\left(x_{0}\right)\right), x_{0}$ is not in the closure of $\pi_{X}\left(g_{f}^{-1}(S)\right)$.

Proof. Note that $(\mu f)^{-1}(N(S))=\left\{x \in X: g_{f}(x, y) \notin S\right.$ for each $\left.y \in Y\right\}=$ $\left\{x \in X:\{x\} \times Y \cap g_{f}^{-1}(S)=\varnothing\right\}=X \mid \pi_{X}\left(g_{f}^{-1}(S)\right)$.

COROllary. Let $C(Y, Z)$ have the topology generated by $\gamma_{a}^{*}$. Then $\mu(C(X \times Y, Z))$ is contained in $C(X, C(Y, Z))$ if and only if $\pi_{X}\left(g_{f}^{-1}(S)\right)$ is closed for each $S \in \gamma_{a}^{*}$ and each $f$ in $C(X \times Y, Z)$.

In a later paper we will consider the remaining exponential laws for topologies generated by functions $\gamma_{a}$, and, indeed, for a more general class. In this paper, where we are primarily interested in closure conditions on projections, we will only prove exponential laws for topologies for which the remaining half of the exponential law is essentially trivial. For fixed spaces $Y, Z$, let $\gamma_{s}^{*}=\{A \times B \subseteq Y \times Z$ : $A$ is the closure of an open set and $B$ is closed $\}$, let $\gamma_{m}^{*}=\{F \subseteq Y \times Z: F$ is a zero set $\}$, let $\gamma_{g}^{*}=\{F \subseteq Y \times Z: F$ is closed $\}$, and letting $R$ denote the real line, define $\gamma_{u}$ by the rule: $\gamma_{u}(f)=\left\{h^{-1}(R \mid(-\varepsilon, \varepsilon)): \varepsilon>0, h=d \circ f \times i, d\right.$ is a continuous pseudometric on $Z$, and $i: Z \rightarrow Z$ is the identity map on $Z$ \}. Of the corresponding topologies, $\mathscr{T}_{s}$ is a set-open topology, $\mathscr{T}_{g}$ is the graph topology of [8], [9] and [10], and $\mathscr{T}_{u}$ is (when $Z$ is completely regular) the topology of uniform convergence. When $Z=R$, and $Y$ is completely regular Hausdorff, $\mathscr{T}_{m}$ is the Moore topology (see for instance [3]) which is usually defined as the topology having a base at $f$ consisting of sets of the form $\{g \in C(X):|g-f|<u\}$ where $u$ is a unit (that is, $\left.u^{-1}(0)=\varnothing\right)$ in $C(X)$. Rewriting this set as $\left\{g: g \cap S=\varnothing, S=h^{-1}([0,+\infty)), h=d \circ(f \times i)-u \circ \pi_{Y}\right\}$ where $d$ is the usual metric on $R, i$ is the identity on $R$ and $\pi_{Y}$ is the projection on 
$Y \times R$, it is apparent that the Moore topology is less than or equal to $\mathscr{T}_{m}$. Equality is established by showing that for any zero set $F \subseteq Y \times R$ with $(Y \times\{0\}) \cap F=\varnothing$, there exists a unit $u$ in $C(Y)$ for which $\{(y, r): 0 \leqq r \leqq u(y)\} \cap F=\varnothing$. This follows from the fact, established in [5], that when $\pi_{Y}$ is $z$-closed and the domain of $\pi_{Y}$ is completely regular Hausdorff, $\pi_{Y}$ maps zero sets to zero sets, and the well known fact that $\pi_{Y}: Y \times Z \rightarrow Y$ is closed for $Z$ compact: Choosing functions $u_{n}$ in $C\left(Y,\left[0,1 / n 2^{n}\right]\right)$ such that $u_{n}^{-1}(0)=\pi_{Y}(Y \times[0,1 / n] \cap F), u=\sum_{n} u_{n}$ is the desired unit.

From the remark above and the manner in which they have been defined, it is clear that $\mathscr{T}_{s} \leqq \mathscr{T}_{g}$ and $\mathscr{T}_{u} \leqq \mathscr{T}_{m} \leqq \mathscr{T}_{g}$; most of these relationships were established in [10] where examples are given which show that each of the topologies are, in general, distinct. Indeed, it is clear that $\mathscr{T}_{u}$ and $\mathscr{T}_{m}$ coincide on the constant functions in $C(Y, Z)$, and in fact have the same neighborhood systems in $C(Y, Z)$ for such functions, if and only if $\pi_{Z}: Y \times Z \rightarrow Z$ is $z$-closed, while $\mathscr{T}_{u}$ and $\mathscr{T}_{g}$ coincide in this manner if and only if $\pi_{Z}$ is closed. When $C(Y, Z)$ is homogeneous in both topologies this implies that they coincide on $C(Y, Z)$.

1.2. THEOREM. Let $Z$ be a topological group. Then on $C(Y, Z), \mathscr{T}_{u}=\mathscr{T}_{m}$ if and only if $\pi_{Z}: Y \times Z \rightarrow Z$ is $z$-closed, and $\mathscr{T}_{u}=\mathscr{T}_{g}$ if and only if $\pi_{Z}$ is closed.

Proof. By the remark above it suffices to prove that translations are continuous in the topologies $\mathscr{T}_{u}, \mathscr{T}_{m}$ and $\mathscr{T}_{g}$ where the group operation of $Z$ is extended pointwise to $C(Y, Z)$. For $\mathscr{T}_{u}$ this is well known (in fact, $\mathscr{T}_{u}$ makes $C(Y, Z)$ a topological group) and for $\mathscr{T}_{m}$ and $\mathscr{T}_{g}$ it follows from the fact that, for $f \in C(Y, Z)$, the map $(y, z) \rightarrow\left(y, z[f(g)]^{-1}\right)$ is a homeomorphism from $Y \times Z$ to $Y \times Z$ which carries $\gamma_{m}(f)$ to $\gamma_{m}(1)$ and $\gamma_{g}(f)$ to $\gamma_{g}(1)$ where 1 denotes the constant function mapping $Y$ to the identity of $Z$.

We might also mention that for arbitrary $Z$ and $Y$ compact Hausdorff, $\mathscr{T}_{u}=\mathscr{T}_{g}$ on $C(Y, Z)$-this is shown in [9]. The following corollary, a consequence of the theorem above and Theorems 3.1 and 2.2 of this paper, generalizes the well-known fact that $\mathscr{T}_{u}=\mathscr{T}_{m}$ on $C(Y)$ if and only if $Y$ is pseudocompact, as well as the fact, established in [10], that $Y$ is countably compact if $\mathscr{T}_{u}=\mathscr{T}_{g}$ on $C(Y)$.

COROLlaRY. Let $Z$ be a nondiscrete locally compact topological group. Then on $C(Y, Z), \mathscr{T}_{u}=\mathscr{T}_{m}$ if and only if $Y$ is pseudocompact, and if $Z$ is also first countable, $\mathscr{T}_{u}=\mathscr{T}_{g}$ if and only if $Y$ is countably compact.

The topology $\mathscr{T}_{s}$ is not, in general, related to $\mathscr{T}_{u}$. In fact, if $\mathscr{T}_{s} \leqq \mathscr{T}_{u}$ or $\mathscr{T}_{s} \geqq \mathscr{T}_{u}$ on $C(Y)$, then $Y$ is pseudocompact. If $f \in C(Y)$ is positive and unbounded, then for $h=d \circ(f \times i), d$ the usual metric on $R, N\left(h^{-1}(R \mid(-1,1))\right.$ is a $\mathscr{T}_{u}$-neighborhood of $f$ which contains no $\mathscr{T}_{s}$-neighborhood of $f$ while $N(Y \times(-\infty, 0])$ is a $\mathscr{T}_{s}$-neighborhood of $1 / f$ which contains no $\mathscr{T}_{u}$-neighborhood of $1 / f$. It follows of course that on $C(Y), \mathscr{T}_{s} \geqq \mathscr{T}_{m}$ only if $\mathscr{T}_{m}=\mathscr{T}_{u}$.

Returning to the exponential laws, the next theorem establishes the remaining inclusion for the topologies $\mathscr{T}_{s}, \mathscr{T}_{u}, \mathscr{T}_{m}$, and $\mathscr{T}_{g}$. 
1.3. THEOREM. Let $X$ be arbitrary and let $\mathscr{T}$ be a topology on $C(Y, Z)$. If $Y$ or $Z$ is regular and $\mathscr{T} \geqq \mathscr{T}_{s}$, or if $Z$ is completely regular and $\mathscr{T} \geqq \mathscr{T}_{u}$, then $\mu(C(X \times Y, Z)$ ) $\supseteq C(X, C(Y, Z))$.

Proof. It suffices to consider the cases $\mathscr{T}=\mathscr{T}_{s}$ with $Y$ or $Z$ regular or $\mathscr{T}=\mathscr{T}_{u}$ with $Z$ completely regular. For the first of these, let $f \in C(X, C(Y, Z))$, let $g=$ $\mu^{-1}(f)$, let $U \subseteq Z$ be open and let $(x, y)$ be any point in $g^{-1}(U)$. By the continuity of $f(x)$ and the regularity of either $Y$ or $Z$, there exists a neighborhood $A$ of $y, A$ the closure of an open set, such that $\{x\} \times A \subseteq g^{-1}(U)$. But then $f^{-1}(N(A \times(Z \mid U))) \times A$ is a neighborhood of $(x, y)$ which is contained in $g^{-1}(U)$, so $g$ is continuous.

For the remaining case, let $f, g, U$, and $(x, y)$ be as before, set $z=g(x, y)$ and note that by the complete regularity of $Z$ there exists a continuous pseudometric $d$ on $Z$ and a real $\varepsilon>0$ such that the $d, \varepsilon$-sphere about $z$ is contained in $U$. Let

$$
\begin{gathered}
V=\left\{y^{\prime} \in Y: d\left(f(x)(y), f(x)\left(y^{\prime}\right)\right)<\varepsilon / 2\right\}, \\
W=f^{-1}\left(N\left(h^{-1}(R \mid(-\varepsilon / 2, \varepsilon / 2))\right)\right)
\end{gathered}
$$

for $h=d \circ(f \times i)$. By the continuity of $f$ and $f(x), W \times V$ is a neighborhood of $(x, y)$. Hence, since $W \times V \subseteq g^{-1}(U), g$ is continuous.

Let II denote the Sierpiński space, that is, the space with two points and three open sets. Under the usual identification of the closed sets in $Y$ with the functions in $C(Y, \mathrm{II}), C(Y, \mathrm{II})$ is the hyperspace (plus the empty subset) of $Y$.

1.4. Theorem. Let $Y$ be regular and $X$ arbitrary. The following conditions on $X \times Y$ are equivalent:

(i) For each space $Z$ and each topology $\mathscr{T}$ on $C(Y, Z)$ satisfying $\mathscr{T}_{s} \leqq \mathscr{T} \leqq \mathscr{T}_{g}$, $(X, Y, Z, \mathscr{T})$ satisfies the exponential law;

(ii) For some topology $\mathscr{T}$ on $C(Y, \mathrm{II})$ satisfying $\mathscr{T}_{s} \leqq \mathscr{T},(X, Y, \mathrm{II}, \mathscr{T})$ satisfies the exponential law;

(iii) $\pi_{X}: X \times Y \rightarrow X$ is closed.

Proof. (i) $\Rightarrow$ (ii) is trivial and (iii) $\Rightarrow$ (i) follows from Corollary 1.1 and Theorem 1.3. (ii) $\Rightarrow$ (iii): We may suppose $\mathscr{T}=\mathscr{T}_{s}$, so by Corollary 1.1 it suffices, given a closed subset $F$ of $X \times Y$, to exhibit $f \in C(X \times Y$, II $)$ and $S$ in $\gamma_{s}^{*}$ such that $g_{f}^{-1}(S)=F$. Let 0 be the closed point in II, let $S=Y \times\{0\}$, and let $f$ be the function with $f^{-1}(0)=F$.

1.5. Theorem. Let $X$ and $Y$ be arbitrary. The following conditions on $X \times Y$ are equivalent:

(i) For each completely regular space $Z$ and each topology $\mathscr{T}$ on $C(Y, Z)$ satisfying $\mathscr{T}_{u} \leqq \mathscr{T} \leqq \mathscr{T}_{m},(X, Y, Z, \mathscr{T})$ satisfies the exponential law.

(ii) For some topology $\mathscr{T}$ on $C(Y)$ satisfying $\mathscr{T}_{u} \leqq \mathscr{T},(X, Y, Z, \mathscr{T})$ satisfies the exponential law.

(iii) $\pi_{X}: X \times Y \rightarrow X$ is $z$-closed. 
Proof. (i) $\Rightarrow$ (ii) is trivial and (iii) $\Rightarrow$ (i) follows from Theorems 1.1 and 1.3. (ii) $\Rightarrow$ (iii): Let $F$ be a zero set in $X \times Y$, say $F=f^{-1}(0)$ and let $x_{0}$ be any point in $X \mid \pi_{X} F$. Define $f^{*}: X \times Y \rightarrow R$ by the rule $f^{*}(x, y)=f(x, y) \mid f\left(x_{0}, y\right)$ and note that $f^{*}$ is continuous since $f\left(x_{0}, y\right)$ is never zero. Since $\mu f^{*}\left(x_{0}\right)=Y \times\{1\}, S=Y \times(R \mid(1 / 2$, $3 / 2))$ is in $\gamma_{u}\left(\mu f^{*}\left(x_{0}\right)\right)$ and hence, by Theorem 1.1, $x_{0}$ is not in the closure of $\pi_{X}\left(g_{f^{*}}^{-1}(S)\right)$. But since $F=g_{f^{*}}^{-1}(Y \times\{0\})$, it follows that $x_{0}$ is not in the closure of $\pi_{X} F$. Therefore $\pi_{X}$ is $z$-closed.

We now give two applications of the theorem above. Recall that a uniform space is fine if its uniformity is the finest (largest) uniformity compatible with its topology, and that fine spaces are characterized by the property: Each continuous function with uniform range is uniformly continuous. The semiuniform product, $X * Y$, of two uniform spaces is the topological space $X \times Y$ with the smallest uniformity making each function in $\mu^{-1}(U(X, U(Y, Z)))$ uniformly continuous, for each uniform space $Z$. $(U(Y, Z)$ is the set of uniformly continuous functions from $Y$ to $Z$ with the uniformity of uniform convergence.)

We will use the facts that $U(X \times Y, Z)$ is always contained in $\mu^{-1}(U(X, U(Y, Z)))$ and that under the obvious identifications, $U(X \times Y, Z)=U(X * Y, Z) \cap U(Y * X, Z)$. (For Hausdorff uniform spaces these are immediate consequences of Theorems 21 and 22, Chapter III of [6]; the proofs hold, without change, for the non-Hausdorff case.)

1.6. TheOREM. Let $X$ and $Y$ be fine. Then $X * Y$ is fine if and only if $\pi_{X}: X \times Y \rightarrow X$ is $z$-closed.

Proof. Identifying functions under the map $\mu$, we have

$$
U(X * Y, Z)=U(X, U(Y, Z))=C(X, C(Y, Z)) \subseteq C(X \times Y, Z),
$$

for any uniform space $Z$. The space $X * Y$ is fine if and only if the final containment is always equality, and by Theorem 1.5 this is true if and only if $\pi_{X}$ is $z$-closed.

1.7. TheOREM. Let $X$ and $Y$ be fine. Then $X \times Y$ is fine if and only if $\pi_{X}$ and $\pi_{Y}$ are both z-closed.

Proof. With the obvious identifications,

$$
U(X * Y, Z)=U(X, U(Y, Z))=C(X, C(Y, Z)) \subseteq C(X \times Y, Z)
$$

and

$$
U(Y * X, Z)=U(Y, U(X, Z))=C(Y, C(X, Z)) \subseteq C(Y \times X, Z)=C(X \times Y, Z) .
$$

The space $X \times Y$ is fine if and only if both containments are equalities, i.e., if and only if $\pi_{X}$ and $\pi_{Y}$ are both $z$-closed.

Our next theorem gives two more equivalences to the condition $\pi_{X}$ is $z$-closed. The relation (i) $\Leftrightarrow$ (iii), for $X$ completely regular Hausdorff, is proved, by different means in [5], and a special case of this result appears as Theorem 3.1 of [2]. Recall that $C^{*}(X)$ denotes the bounded functions in $C(X)$ and that a subset $X^{\prime}$ of $X$ is said 
to be $C^{*}$-embedded in $X$ if each function in $C^{*}\left(X^{\prime}\right)$ admits a continuous extension to $X$. Let $C^{*}\left(X, C^{*}(Y)\right)$ denote the set of functions, $f$, in $C(X, C(Y))$ such that $\bigcup_{x \in X} f(x)(Y)$ is bounded.

1.8. THEOREM. For $X$ arbitrary and $Y$ completely regular Hausdorff, and where $C^{*}(Y)$ has the topology $\mathscr{T}_{u}$, the following conditions are equivalent:

(i) $\pi_{X}: X \times Y \rightarrow X$ is $z$-closed;

(ii) $\mu\left(C^{*}(X \times Y)\right)=C^{*}\left(X, C^{*}(Y)\right)$;

(iii) $X \times Y$ is $C^{*}$-embedded in $X \times \beta Y$.

Proof. (i) $\Rightarrow$ (ii). By $1.5, \mu(C(X \times Y))=C(X, C(Y))$; clearly $\mu$ carries $C^{*}(X \times Y)$ onto $C^{*}\left(X, C^{*}(Y)\right)$.

(ii) $\Rightarrow$ (i) follows from the proof of (ii) $\Rightarrow$ (iii) in Theorem 1.5 since each zero set in $X \times Y$ is the zero set of a bounded function.

(ii) $\Rightarrow$ (iii). Since $C^{*}(Y)$ is homeomorphic to $C(\beta Y)$,

$$
C^{*}\left(X, C^{*}(Y)\right)=C^{*}(X, C(\beta Y))
$$

under the obvious identification. But, since $\beta Y$ is compact, $\pi: X \times \beta Y \rightarrow X$ is closed (this well-known fact follows from Theorem 2.2 in the next section) so by Theorem $1.5 C^{*}(X, C(\beta Y))$ and hence $C^{*}(X \times Y)$ is contained in $C(X \times \beta Y)$.

(iii) $\Rightarrow$ (ii). Let $f \in C^{*}(X \times Y)$, let $f^{\prime}$ be the extension of $f$ in $C(X \times \beta Y)$ and note that $\mu^{-1}\left(f^{\prime}\right)$ is in $C^{*}(X, C(\beta Y))$ so $\mu(f)$ is in $C^{*}\left(X, C^{*}(Y)\right)$. Thus $\mu\left(C^{*}(X \times Y)\right)$ $\subseteq C^{*}\left(X, C^{*}(Y)\right)$ and the reverse inclusion follows from Theorem 1.3.

From Theorems 1.6 and 1.8 it follows that $X * Y$ is fine if and only if $X \times Y$ is $C^{*}$-embedded in $X \times \beta Y$; this is Theorem 39, Chapter VII of [6].

2. Closed projections. In this section we consider conditions necessary or sufficient that a projection be closed. Our first theorem disposes of the trivial case where one of the factors is discrete. For $\mathfrak{n}$ an infinite cardinal, a space is $\mathfrak{n}$-discrete if each $\mathfrak{n}$-fold intersection of open sets is open. A space which is $\mathfrak{m}$-discrete for each $\mathfrak{m}<\mathfrak{n}$ will be called $<\mathfrak{n}$-discrete.

2.1. THEOREM. If $X$ is discrete, then $\pi_{X}$ is closed. If $Y$ is discrete, then $\pi_{X}$ is closed (or if $X$ is completely regular, $\pi_{X}$ is $z$-closed) if and only if $X$ is $\mathfrak{n}$-discrete for $\mathfrak{n}=$ card $Y$.

Proof. That $\pi_{X}$ is closed when $X$ is discrete is clear. If card $Y=\mathfrak{n}$ and $X$ is $\mathfrak{n}$-discrete, then $\pi_{X}$ is closed (and hence $z$-closed) since for $H \subseteq X \times Y$ closed, $\pi_{X} H=\bigcup_{y \in Y} \pi_{X}((X \times\{y\}) \cap H)$ is, like any $\mathfrak{n}$-fold union of closed subsets of $X$, closed. On the other hand, if $\pi_{X}$ is closed, $Y$ discrete, and card $Y=\mathfrak{n}$, then any $\mathfrak{n}$-fold union of closed subsets of $X$, being $\pi_{X} H$ for a closed subset $H$ of $X \times Y$, is closed, so $X$ is $\mathfrak{n}$-discrete. Similarly, if $\pi_{X}$ is $z$-closed, $Y$ discrete and card $Y=\mathfrak{n}$, any $\mathfrak{n}$-fold union of zero sets of $X$ must be closed; when $X$ is completely regular this shows that $X$ is n-discrete. 
We call a space $\mathfrak{m}-\mathfrak{n}$-compact, $\mathfrak{m}$ and $\mathfrak{n}$ infinite cardinals, if each open cover of cardinality $\mathfrak{m}$ admits a subcover of cardinality less than $\mathfrak{n}$; spaces which are $\mathfrak{m}$-n-compact for each $\mathfrak{m} \geqq \mathfrak{n}$ are called $\mathfrak{n}$-compact. Thus $\boldsymbol{\aleph}_{0}-\boldsymbol{\aleph}_{0}$-compact $=$ countably compact, $\aleph_{0}$-compact $=$ compact, and $\aleph_{1}$-compact $=$ Lindelöf. For a cardinal $\mathfrak{n}$ let $W(\mathfrak{n})$ denote the least ordinal of cardinality $\mathfrak{n}$. We say that a point $x$ in $X$ is of type $\mathfrak{m}-\mathfrak{n}$, for infinite cardinals $\mathfrak{m}$ and $\mathfrak{n}$, if $\mathfrak{m}$ is the smallest cardinal of a neighborhood base at $x$ and there exists a family $\left\{S_{\alpha}: \alpha \in W(\mathfrak{n})\right\}$ of closed subsets of $X$ such that $x$ is in the closure of $\bigcup_{\alpha \in W(n)} S_{\alpha}$ but is not in the closure of $\bigcup_{\alpha<\alpha_{0}} S_{\alpha}$ for any $\alpha_{0}$ in $W(\mathfrak{n})$.

2.2. THEOREM. If $Y$ is $\mathfrak{m}-\mathfrak{n}$-compact whenever $X$ contains a point of type $\mathfrak{m}-\mathfrak{n}$, then $\pi_{X}: X \times Y \rightarrow X$ is closed. If $\pi_{X}$ is closed and, for some $\mathfrak{m}, X$ contains a point of type $\mathfrak{m}-\mathfrak{n}$, then $Y$ is $\mathfrak{n}-\mathfrak{n}$-compact.

Proof. Suppose $Y$ is $\mathfrak{m}$-n-compact whenever $X$ contains a point of type $\mathfrak{m}-\mathfrak{n}$, let $H \subseteq X \times Y$ be closed and suppose $x$ is in the closure of $\pi_{X} H$ but is not in $\pi_{X} H$. Let $\left\{U_{\alpha}: \alpha \in W(\mathfrak{m})\right\}$ be a neighborhood base for $x$ of minimum cardinality and set $S_{\alpha}=\operatorname{cl}\left(X \mid U_{\alpha}\right) \cap \pi_{X} H$. Since $x$ is in the closure of the union of the $S_{\alpha}$, there exists a smallest cardinal, $\mathfrak{n}$, such that $x$ is in the closure of some $\mathfrak{n}$-fold union of the $S_{\alpha}$.

Since $x$ is in the closure of $\pi_{X} H$ but is not in $\pi_{X} H, x$ is not isolated and hence $m$ is infinite. Also, $\mathfrak{n}$ is infinite: If it were finite $\pi_{X} H$ would contain some point, $x^{\prime}$, which would be in each neighborhood of $x$; but then, for $y$ such that $\left(x^{\prime}, y\right)$ was in $H,(x, y)$ would be in the closure of $\left(x^{\prime}, y\right)$, so $(x, y)$ would be in $H$ and hence $x$ would be in $\pi_{X} H$. Thus $x$ is of type $\mathfrak{m}-\mathfrak{n}$, and therefore $Y$ is $\mathfrak{m}$-n-compact.

For each $\alpha \in W(\mathfrak{m})$ let $V_{\alpha}$ be the interior of $\left\{y \in Y:\left(U_{\alpha} \times\{y\}\right) \cap H=\varnothing\right\}$ and note that $\left\{V_{\alpha}: \alpha \in W(\mathfrak{m})\right\}$ is an open cover of $Y$, so there exists a subcover $\left\{V_{\alpha}: \alpha \in W^{\prime}\right\}$ where $W^{\prime}$ has cardinality less than $\mathfrak{n}$. But $x$ is not in the closure of $\bigcup_{\alpha \in W^{\prime}} S_{\alpha}$, so $U=X \mid \operatorname{cl}\left(\bigcup_{\alpha \in W^{\prime}} S_{\alpha}\right)$ is a neighborhood of $x$ which, since $U \subseteq \bigcap_{\alpha \in W^{\prime}} U_{\alpha}$, does not meet $\pi_{X} H$. This contradicts our assumption that $x$ is in the closure of $\pi_{X} H$, so $\pi_{X}$ is closed.

Now suppose that $\pi_{X}$ is closed, let $x$ be a point in $X$ of type $\mathfrak{m}-\mathfrak{n}$, and let $\left\{S_{\alpha}: \alpha \in W(\mathfrak{n})\right\}$ be closed sets for which $x$ is in the closure of $\bigcup_{\alpha \in W(\mathfrak{n})} S_{\alpha}$ but is not in the closure of $\bigcup_{\alpha<\alpha_{0}} S_{\alpha}$ for any $\alpha_{0}$ in $W(\mathfrak{n})$. Suppose $Y$ is not $\mathfrak{n}$-n-compact and let $\left\{F_{\alpha}: \alpha \in W(\mathfrak{n})\right\}$ be a collection of nonempty closed subsets of $Y$ such that $\bigcap_{\alpha \in W(\mathfrak{n})} F_{\alpha}=\varnothing, \alpha>\beta \Rightarrow F_{\alpha} \subseteq F_{\beta}$. Let $H$ be the closure of $\bigcup_{\alpha \in W(\mathfrak{n})} S_{\alpha} \times F_{\alpha}$. For any $y$ in $Y$ there exists an $\alpha_{0}$ in $W(\mathfrak{n})$ such that $y \notin F_{\alpha_{0}}$, and there exists a neighborhood $U$ of $x$ such that $U \cap \bigcup_{\alpha<\alpha_{0}} S_{\alpha}=\varnothing$, so $(x, y)$ is not in $H$. Thus $x$ is not in $\pi_{X} H$, but since $x$ is in $\operatorname{cl}\left(\pi_{X} H\right)=\operatorname{cl}\left(\bigcup_{\alpha \in W(\mathfrak{n})} S_{\alpha}\right)$, this contradicts our assumption that $\pi_{X}$ is closed.

For a space $X$ let $b(X)$ be the least cardinal such that each point in $X$ has an open neighborhood base of cardinality less than or equal to $b(X)$. Various special cases of the following corollaries are contained in [2], [5] and [7]. Note that the requirement that $\mathfrak{n}$ be regular which appears in 2.3 and 2.5 is not very restrictive. If $\mathfrak{n}$ is not 
regular and $X$ is $<\mathfrak{n}$-discrete, then $X$ is $\mathfrak{n}$-discrete and hence $<\mathfrak{n}^{\prime}$-discrete for $\mathfrak{n}^{\prime}$ the successor of $\mathfrak{n}$. Of course, $\mathfrak{n}^{\prime}$ is regular.

2.3. Corollary. For a fixed space $X, \pi_{X}$ is closed for each $\mathfrak{n}$-compact space $Y$ if and only if $X$ is $<\mathfrak{n}$-discrete; and if $\mathfrak{n}$ is regular $\pi_{Y}$ is closed for each $<\mathfrak{n}$-discrete space $Y$ if and only if $X$ is $\mathfrak{n}$-compact.

2.4. Corollary. For a fixed space $X, \pi_{X}$ is closed for each $n-\aleph_{0}$-compact space $Y$ if $b(X) \leqq \mathfrak{n}$; and $\pi_{Y}$ is closed for each space $Y$ having $b(Y) \leqq \mathfrak{n}$ if and only if $X$ is $\mathfrak{n}-\aleph_{0}$-compact.

2.5. Corollary. For a fixed space $X, \pi_{X}$ is closed for each $\mathfrak{n}$-n-compact space $Y$ if $X$ is $<\mathfrak{n}$-discrete with $b(X) \leqq \mathfrak{n}$; and for $\mathfrak{n}$ regular $\pi_{Y}$ is closed for each $<\mathfrak{n}$ discrete space $Y$ with $b(Y) \leqq \mathfrak{n}$ if and only if $X$ is $\mathfrak{n}$-n-compact.

2.6. Corollary. There is no space $X$ for which $\pi_{X}$ is closed if and only if $Y$ is $\mathfrak{n}$-compact; if $X$ is $<\mathfrak{n}$-discrete and $b(X)=\mathfrak{n}$, then $\pi_{X}$ is closed if and only if $Y$ is $\mathfrak{n}$-n-compact.

The following two examples show that neither of the conditions in 2.2 is both necessary and sufficient that $\pi_{X}$ be closed. The second example, and the proofs of both examples were supplied by A. Hager in a letter dated November 14, 1967. (The proofs are adapted from the proof of a much more general result of Hager's which gives necessary and sufficient conditions on $X$ that $\pi_{X}: X \times Y \rightarrow X$ be closed for each space $Y$ having a given fixed cardinality.)

2.7. ExAMPLE. There exist spaces $X$ and $Y$ where each point of $X$ is isolated or of type $c-\aleph_{0}, Y$ is not $c-\aleph_{0}$-compact (or even $\aleph_{1}-\aleph_{0}$-compact) and $\pi_{X}: X \times Y \rightarrow X$ is closed.

Proof. For $N^{*}=N \cup\{\infty\}$ the one-point compactification of the integers let $X$ be the quotient of $N^{*} \times N$ formed by identifying the points $(\infty, n)$ and note that the nonisolated point of $X$ is of type $c-\aleph_{0}$. Let $Y=W\left(\aleph_{1}\right)$ with the order topology. Since $Y$ is not compact, it is not $\boldsymbol{\aleph}_{1}-\boldsymbol{\aleph}_{0}$-compact and thus is not $c$ - $\boldsymbol{\aleph}_{0}$-compact. To see that $\pi_{X}: X \times Y \rightarrow X$ is closed, let $F \subseteq X \times Y$ be closed, index $\pi_{X} F=\left\{x_{n}\right\}$, choose $y_{n}$ in $Y$ such that $\left(x_{n}, y_{n}\right)$ is in $F$, set $K=\operatorname{cl}\left\{y_{n}\right\}, F^{\prime}=(X \times K) \cap F$ and note that $\pi_{X} F=\pi_{X} F^{\prime}$ is closed since $K$ is compact.

2.8. ExAMPLE. There exist spaces $X$ and $Y$ where each point in $X$ is isolated or of type $c-\aleph_{0}$ and $Y$ is countably compact but $\pi_{X}: X \times Y \rightarrow X$ is not closed.

Proof. For $N$ the positive integers, let $p$ be a $P$-point of $\beta N \backslash N$ and note that $Y=\beta N \mid\{p\}$ is countably compact (such a $p$ exists by $9 \mathrm{M}$ of [3]). Set $X=N \cup\{p\}$ with the relative topology. Then $p$ is of type $c-\aleph_{0}$, but $\pi_{X}$ is not closed:

$$
\Delta=\{(n, n): n \in N\} \leqq X \times Y
$$

is closed while $\pi_{X}(\Delta)=N$ is not.

3. z-closed projections. We now consider conditions under which projections will be $z$-closed. For this reason, we assume throughout this section that $X$ and $Y$ 
are completely regular, though not necessarily Hausdorff. Note that the case where one of the factors is discrete is already covered by 2.1 ; for the general case we need the following concept: A space $X$ is pseudo-n-compact if each discrete family of open subsets of $X$ has cardinality less than $\mathfrak{n}$. (Recall that a family of subsets of $X$ is discrete if each point of $X$ has a neighborhood which meets at most one of the members of the family.) By Theorem 30, Chapter VII of [6], and the fact that in a fine space each discrete family of open sets is uniformly discrete, the definition of pseudo-n-compactness given above is equivalent to the definition given in [6], and pseudo- $\aleph_{0}$-compact $=$ pseudocompact.

3.1. TheOREM. Suppose neither $X$ nor $Y$ is discrete. If $\pi_{X}: X \times Y \rightarrow X$ is $z$-closed, then for each cardinal $\mathfrak{n}$ either $X$ is $\mathfrak{n}$-discrete or $Y$ is pseudo-n-compact.

Proof. Suppose not. Let $S=\bigcup_{\alpha \in W(\mathfrak{n})} S_{\alpha}$ where each $S_{\alpha} \subseteq X$ is closed, $\beta<\alpha$ $\Rightarrow S_{\alpha} \leqq S_{\beta}$ and $x_{0} \in \operatorname{cl} S \mid S$, and let $\left\{U_{\alpha}: \alpha \in W(\alpha)\right\}$ be a discrete family of open nonempty subsets of $Y$. Choosing points $y_{\alpha} \in U_{\alpha}$, let $f_{\alpha}^{1}: Y \rightarrow[0,1]$ be a continuous function mapping $y_{\alpha}$ to 1 and $Y \backslash U_{\alpha}$ to 0 , and let $f_{\alpha}^{2}: X \rightarrow[0,1]$ be a continuous function taking $x_{0}$ to 0 and $S_{\alpha}$ to $\{1\}$. Define $f: X \times Y \rightarrow R$ by the relation:

$$
\begin{aligned}
f(x, y) & =f_{\alpha}^{1}(x) f_{\alpha}^{2}(y) & & \text { if } y \in U_{\alpha}(\text { for some } \alpha) \\
& =0 & & \text { otherwise }
\end{aligned}
$$

and note that $f$ is continuous. Now $\bigcup_{\alpha}\left(S_{\alpha} \times\left\{y_{\alpha}\right\}\right) \subseteq f^{-1}(1)$, and so $S \subseteq \pi_{X} f^{-1}(1)$, but $x_{0} \notin \pi_{X} f^{-1}(1)$ which contradicts the assumption that $\pi_{X}$ is $z$-closed.

3.2. CoRollary. If $\pi_{X}$ and $\pi_{Y}$ are closed, and neither $X$ nor $Y$ is discrete, then there exists a cardinal $\mathfrak{n}$ such that $X$ and $Y$ are pseudo-n-compact and $<\mathfrak{n}$-discrete.

Proof. By Theorem 3.1, for $\mathfrak{n}_{1}$ the smallest cardinal such that $X$ is not $\mathfrak{n}_{1-}$ discrete and $\mathfrak{n}_{2}$ the smallest cardinal such that $Y$ is not $\mathfrak{n}_{2}$-discrete, $Y$ is pseudo- $\mathfrak{n}_{1}$ compact and $X$ is pseudo- $\mathfrak{n}_{2}$-compact. Since an $\mathfrak{n}$-discrete pseudo-n-compact space is discrete, and since neither $X$ nor $Y$ is discrete, we must have $\mathfrak{n}_{1} \leqq \mathfrak{n}_{2}$ and $\mathfrak{n}_{2} \leqq \mathfrak{n}_{1}$, so $\mathfrak{n}=\mathfrak{n}_{1}=\mathfrak{n}_{2}$ is the desired cardinal.

3.3. Lemma. Let $X$ be $<\mathfrak{n}$-discrete, card $X \geqq \mathfrak{n}$, and suppose $X \times Y$ is not pseudo$\mathfrak{n}$-compact. Then there exists a discrete family $\left\{U_{\alpha} \times V_{\alpha}: \alpha \in W(\mathfrak{n})\right\}$ of nonempty open subsets of $X \times Y$ with the $U_{\alpha}$ disjoint.

Proof. We first note that $X$ contains $\mathfrak{n}$ disjoint open subsets. If $X$ is discrete this is obvious, and for $x_{0} \in X$ not isolated there exists a decreasing family $\left\{W_{\alpha}: \alpha\right.$ $\in W(\mathfrak{n})\}$ of neighborhoods of $X_{0}$ having $W_{\alpha} \mid \operatorname{cl} W_{\alpha+1} \neq \varnothing$ for each $\alpha$. If $Y$ is not pseudo-n-compact we may complete the proof by taking $\left\{V_{\alpha}\right\}$ discrete and $\left\{U_{\alpha}\right\}$ disjoint, so we assume that $Y$ is pseudo-n-compact. Let $\left\{U_{\alpha}^{\prime} \times V_{\alpha}^{\prime}: \alpha \in W(\mathfrak{n})\right\}$ be a discrete family of nonempty open subsets of $X \times Y$ and set $X^{\prime}=\cup_{\alpha} U_{\alpha}^{\prime}$. Since $X^{\prime}$ is open in $X$, we may assume that fewer than $\mathfrak{n}$ of the points of $X^{\prime}$ are isolated (in $X^{\prime}$ ); thus, since $X^{\prime} \times Y$ would be pseudo-n-compact if card $X^{\prime}$ were less than $\mathfrak{n}, X^{\prime}$ 
contains a point $x_{0}$ which is not isolated. Take $\left\{W_{\alpha}: \alpha \in W(\mathfrak{n})\right\}$ as above and choose $\gamma(\alpha)$ such that

$$
U_{\alpha}=U_{\gamma(\alpha)}^{\prime} \cap\left(W_{\alpha} \mid \mathrm{cl} W_{\alpha+1}\right) \neq \varnothing .
$$

The family $\left\{U_{\alpha} \times V_{y(\alpha)}^{\prime}: \alpha \in W(\mathfrak{n})\right\}$ has the desired properties.

The case $\mathfrak{n}=\boldsymbol{N}_{0}$ of the lemma above is of course well known. The case $\mathfrak{n}=\boldsymbol{N}_{0}$ of the following theorem is almost equivalent to a result due to Tamano in [6]. As part of his Theorem 1 he shows (by a proof different from ours) that for $X$ and $Y$ infinite, $\beta(X \times Y)=\beta X \times \beta Y$ (i.e., $X \times Y$ is pseudocompact) if and only if $X$ and $Y$ are pseudocompact and $\pi_{X}$ is $z$-closed.

3.4. TheOrem. For $X$ and $Y<n$-discrete but not discrete, the following conditions on $X \times Y$ are equivalent :

(i) $X$ is pseudo-n-compact and $\pi_{X}$ is $z$-closed;

(ii) $X \times Y$ is pseudo-n-compact;

(iii) $Y$ is pseudo-n-compact and $\pi_{Y}$ is $z$-closed;

(iv) $\pi_{X}$ and $\pi_{Y}$ are $z$-closed and $X($ or $Y)$ is not $\mathfrak{n}$-discrete.

Proof. By Corollary 3.2 it suffices to establish (i) $\Leftrightarrow$ (ii).

(i) $\Rightarrow$ (ii). Suppose $X \times Y$ is not pseudo-n-compact, let $\left\{U_{\alpha} \times V_{\alpha}: \alpha \in W(\mathfrak{n})\right\}$ be a discrete family as in 3.3 , choose zero sets $W_{\alpha} \subseteq U_{\alpha} \times V_{\alpha}, W_{\alpha}$ having nonempty interior. Then $\bigcup_{\alpha} W_{\alpha}$ is a zero set so $\pi_{X}\left(\bigcup_{\alpha} W_{\alpha}\right)$ is closed. But this implies that \{int $\left.\left(\pi_{X} W_{\alpha}\right): \alpha \in W(\mathfrak{n})\right\}$ is a discrete family, contrary to the assumption that $X$ is pseudo-n-compact.

(ii) $\Rightarrow$ (i). First suppose $\mathfrak{n}=\boldsymbol{\aleph}_{0}$. By Glicksberg's Theorem (Theorem 1 of [4]) $C(X \times Y)=C(\beta X \times \beta Y)$. Since $C(Y)$ and $C(\beta Y)$ are homeomorphic (because $Y$ is pseudocompact), since $C(\beta Y)$ is complete and since $\beta X$ is the topological completion of $X$ (because $X$ is pseudocompact), we have $C(X, C(Y))=C(X, C(\beta Y))$ $=C(\beta X, C(\beta Y))$ under the obvious identifications. But by Theorems 2.2 and 1.2, $C(\beta X, C(\beta Y))=C(\beta X \times \beta Y)$, so $C(X \times Y)=C(X, C(Y))$. Thus by Theorem 1.3, $\pi_{X}$ is $z$-closed.

Now suppose $\mathfrak{n}>\boldsymbol{\aleph}_{0}$, so $X$ has a base of clopen sets, and zero sets in $X \times Y$ are open. Let $H$ be any zero set in $X \times Y$, and write the open set $\pi_{X} H$ as a disjoint union $\left\{H_{\alpha}: \alpha \in A\right\}$ of nonempty clopen subsets. Since $H$ is clopen, $\left\{\left(H_{\alpha} \times Y\right)\right.$ $\cap H: \alpha \in A\}$ is a discrete family of nonempty open subsets of $X \times Y$, and hence card $(A)<\mathfrak{n}$. Therefore $H$, being a union of fewer than $\mathfrak{n}$ closed sets, is closed.

In [2] Comfort and Negrepontis define and study the following notion: A pair of completely regular Hausdorff spaces is a $C^{*}$-pair if $X \times Y$ is $C^{*}$-embedded in both $X \times \beta Y$ and $\beta X \times Y$. Combining Theorems 3.4, 2.1, 1.6 and 1.5, we have:

3.5. THEOREM. For completely regular Hausdorff spaces $X$ and $Y$, the following conditions are equivalent:

(i) $\pi_{X}$ and $\pi_{Y}$ are $z$-closed;

(ii) $(X, Y)$ is a $C^{*}$-pair; 
(iii) When $X$ and $Y$ are given their fine uniformities, $X \times Y$ is fine;

(iv) Either one of the spaces $X, Y$ is discrete of cardinality $\mathfrak{n}$ while the other is $\mathfrak{n}$-discrete; or there exists a cardinal $\mathfrak{n}$ such that $X \times Y$ is pseudo-n-discrete and $<\mathfrak{n}$ discrete.

The equivalence of (i) with (ii) is due to A. W. Hager, and the equivalence of (iii) with (iv) is Isbell's characterization of fine products. The equivalence of (ii) with (iv) is new and can be used to unify and generalize many of the results of [2]. For instance, it follows from Theorems 3.4 and 2.1 that $(X, Y)$ is a $C^{*}$-pair for each $\mathfrak{n}$-discrete space $Y$ with card $(Y) \geqq \mathfrak{n}$ if and only if $X$ is discrete of cardinality at most $\mathfrak{n}$. This generalizes Theorem 4.2 of [2] as well as the conjecture in 4.3 of [2] which was proved by A. W. Hager, these being essentially the case $\mathfrak{n}=\boldsymbol{N}_{0}$.

\section{REFERENCES}

1. W. W. Comfort, On the Hewitt realcompactification of a product space, Trans. Amer. Math. Soc. 131 (1968), 107-118.

2. W. W. Comfort and S. Negrepontis, Extending continuous functions on $X \times Y$ to subsets of $\beta X \times \beta Y$, Fund. Math. 59 (1966), 1-12.

3. L. Gillman and M. Jerison, Rings of continuous functions, Van Nostrand, New York, 1960.

4. I. Glicksberg, Stone-Cech compactifications of products, Trans. Amer. Math. Soc. 90 (1959), 369-382.

5. A. W. Hager and S. Mrowka, Unpublished manuscript.

6. J. R. Isbell, Uniform spaces, Math. Surveys, No. 12, Amer. Math. Soc., Providence, R. I., 1964.

7. S. B. Nadler, Jr., A note on projections and compactness, Abstract $617-19$, Notices Amer. Math. Soc. 11 (1964), 753-754.

8. S. A. Naimpally, Graph topology for function spaces, Trans. Amer. Math. Soc. 123 (1966), 267-272.

9. S. A. Naimpally and C. M. Pareek, Graph topology for function spaces. II, (to appear).

10. H. Poppe, Uber Graphentopologien für Abbildungsräume. I, Bull. Acad. Polon. Sci. 15 (1967), 71-80.

11. H. Tamano, A note on the pseudo-compactness of the product of two spaces, Mem. Coll. Sci. Univ. Kyoto Ser. A Math. 33 (1960/61), 225-230.

Clark University,

WORCESTER, MASSACHUSETTS 